\title{
Effects of size-brightness and complexity of non-meaningful stimulus material on EEG desynchronization'
}

\author{
DAYID GIBSON, GARRY BAKER AND ELAINE RATHIE \\ THE UNIVERSITY OF CAL'GARY
}

Latency and duration of EEG desynchronization were measured while Ss were exposed to nine slides varying in complexity and size-brightness. There was a positive relationship between duration of EEG desynchronization and the simple stimulus impact va iable but no effect due to nonmeaningful complexity levels. There was no relation between duration of latency period and either stimulus complexity or sizebrightness. The results were evaluated in the context of the "collative variables" hypothesis and the alerting scanning, and integrative postulates for EEG desynchronization phenomena.

Debate about the significance of EEG desynchronization as a product of external stimuli has revolved about the alerting-orientation hypothesis (Bishop, 1933), the scanning hypothesis (Walters, 1953), and the integrative hypothesis (Klein \& Krech, 1952). Stimulus material properties governing maintanence of EEG desynchronization are less well studied than stimulus characteristics regulating onset of alpha block. Baker \& Franken (1967) demonstrated a positive relation between alpha recovery time and stimulus complexity level but found no effect due to stimulus impact characteristics (size and brightness). The results are seen to support Berlyne \& McDonnell's (1956) stimulus complexity interpretation for duration of EEG desynchronization. Complexity is said to increase with number of distinguishable elements and/or dissimilarities between elements (Berlyne, 1966). While Baker \& Franken and Berlyne \& McDonnell showed that "amount of material" influenced duration of EEG desynchronization, it is not clear from these studies whether simple stimulus intensity variables or information content variables were being manipulated. Both studies employed levels of complexity based on symbolically meaningful stimulus material. The present study examined duration of EEG desynchronization in response to three stimulus intensity levels with three complexity gradients comprised of information-free stimulus materials.

\section{Subjects}

Fifteen students from the University of Calgary were randomly selected within the limits of displaying more than $25 \%$ or less than $90 \%$ alpha over the recording time of the experiment.

Apparatus

A Grass Model 7 polygraph was used to record EEG on one channel with a 7P3A preamplifier and a 7DAB driver amplifler. A second channel marked time in sec (paper speed was $25 \mathrm{~mm}$ per sec), while a third registered the beginning and end of each stimulus pattern presentation. A monopolar recording was taken from the left occipital lobe of each $\mathrm{S}$ by means of a single silver disc electrode. There was a reference electrode on the corresponding ear and a ground electrode attached to the arm.

The $S$ was placed in a reclining position facing a blank wall in a darkened room. The $E$ and apparatus were in an adjoining room from which the $S$ could be viewed through a one-way window. A $35 \mathrm{~mm}$ Kodak Carousel 800 projector was used to present the slides. Each slide was presented for $3 \mathrm{sec}$ with an interval of 17 sec.

Stimulus slides. Nine negattve (white on black) stimulus slides were graduated by three degrees of complexity and three levels of size. The smallest size occupied $25 \%$ of the projection surface, the middle size $50 \%$, and the largest slide $100 \%$. The least complex design comprised three straight lines of equal length arranged in random drop-stick fashion. The middle level of complexity engaged six lines of random arrangement, and so on. The "designs" displayed no harmonious relattonship and afforded limited opportunity for cognitive association by Ss. This impression is confirmed by Ss' reports. A second series of slides involving syllables and designs was shown prior to the experimental slides in order to habituate Ss to slide projector sounds and presentation rate.

\section{Procedure}

Ss were assured of the benign nature of the experiment after positioning and electrode placement were complete. Relaxed and passive $S$ participation was encouraged. Instrument calibration was completed prior to exposure of the habituation slides. A $30 \mathrm{sec}$ delay followed showing of the habituation slides whereupon nine size-complexdty combinations were presented in randomized order for 3 sec each with a 17 sec interval.

Scoring. EEG scoring procedures were similar to that used by Berlyne \& McDonnell and Wilson \& Wilson (1959). A desynchronization was scored when the amplitude of the alpha record dropped below $50 \%$ or the dominant frequency became greater than 13 cps. A return of three consecutive alpha waves was considered a recovery. If no recovery was noted, a full score of $19 \mathrm{sec}$ was given. The full $20 \mathrm{sec}$ required for a complete sequence was not given because the noise of 
the apparatus changing slides began 1 sec before the slide actually appeared. The score recorded was the length of time in sec from the disappearance to the return of the alpha rhythm.

\section{Results and Discussion}

Subjects by treatments analysis of the data (MCNemar, 1962) was made for recovery time (duration of EEG desynchronization) and for latency time. The size variable was effective for recovery time $(F=4.36$, df $=2 / 30, p<.05)$. The complexity variable had no significant effect on length of desynchronization (F $=1.65, \mathrm{df}=2 / 30$ ). There were no significant effects of size-brightness or complexity with latency time. There was a significant difference in alpha recovery time between the small and large slides $(d=1.86$, $\mathrm{p}<.05)$, irrespective of complexity level, but not between the small and medium slides or between the medium and large slides. Recovery time for the medium slides fell between the large and small slides.

The results of the present study showed that more prolonged EEG desynchronization occurred in response to stimulus size-brightness properties than to stimulus complexity levels. The differences in findings between the Baker and Franken study and the present investigation support Berlyne's contention that when complex stimulus patterns have a relatively higher information content, EEG desynchronization will be as protracted as required for optimum information retrieval. Otherwise, complexity level of stimulus material, devoid of epistemic potential, does not apparently influence duration of alpha desynchronization. Quite simple patterns having high visual impact elicited longer alpha block than the more complex designs with less visual impact. The present results do not support the collative variables concept that stimulus complexity as such is invested with special drive properties, or that the differential visual scanning potential of nonmeaningful stimulus complexity levels is reflected in duration of EEG desynchronization. Future studies might attempt to distinguish between the arousal, or orienting properties of the stimulus and the cognitive potential of the stimulus material. Further examination should be made of both stimulus and subject variables governing the interrelation of stimulus meaningfulness characteristics, stimulus intensity levels, and duration of EEG desynchronization.

\section{Reterences}

Baker, G., \& Franken, R. Effects of stimulus size, brightness and complexity upon EEG desynchronization. Psychon. Sci.. 1967, 7, 289-290.

Berlyne, n. E. Motivational problems raised by exploratory and epistemic behavior. In S. Koch (Ed.), Psychology: A study of a Science. New York: McGraw-Hill, 1963. Pp. 284-364.

Berlyne, D. E. Structure and direction in thinking. New York: Wiley, 1966.

Berlyne, D. E., \& McDonnell, P. Effects of stimulus complexity on duration of EEG desynchronization. EEG clin. Neurophysiol. , $1965,18,156-161$.

Bishop, G. H. Cyclic changes in excitahility of the optic pathway of the rabbit. Amer. J. Physiol., 1933, 103, 213-224.

Klein, G. S., \& Krech, D. Cortical conductivity in the brain-injured. J. Pers., 1952, 21, 118-148.

McNemar, Q. Psycholonical statistics. New York: Wiley, 1962. Walters, w. G. The living brain. New York: Norton 1953.

Wilson, J. N., \& Wilson, W. P. The duration of human EEG arousal responses elicited by photic stimulation. EEG clin. Neurophysiol., 1959, 11, 85-91.

\section{Hote}

1. Supported in part by N. R. C. Grant (Canada) APA-220. 